\title{
SEMILINEAR PROBLEMS WITH BOUNDED NONLINEAR TERM
}

MARTIN SCHECHTER

Received 17 August 2004

We solve boundary value problems for elliptic semilinear equations in which no asymptotic behavior is prescribed for the nonlinear term.

\section{Introduction}

Many authors (beginning with Landesman and Lazer [1]) have studied resonance problems for semilinear elliptic partial differential equations of the form

$$
-\Delta u-\lambda_{\ell} u=f(x, u) \quad \text { in } \Omega, \quad u=0 \quad \text { on } \partial \Omega,
$$

where $\Omega$ is a smooth bounded domain in $\mathbb{R}^{n}, \lambda_{\ell}$ is an eigenvalue of the linear problem

$$
-\Delta u=\lambda u \quad \text { in } \Omega, \quad u=0 \quad \text { on } \partial \Omega,
$$

and $f(x, t)$ is a bounded Carathéodory function on $\Omega \times \mathbb{R}$ such that

$$
f(x, t) \longrightarrow f_{ \pm}(x) \quad \text { a.e. as } t \longrightarrow \pm \infty \text {. }
$$

Sufficient conditions were given on the functions $f_{ \pm}$to guarantee the existence of a solution of (1.1). (Some of the references are listed in the bibliography. They mention other authors as well.)

In the present paper, we consider the situation in which (1.3) does not hold. In fact, we do not require any knowledge of the asymptotic behavior of $f(x, t)$ as $|t| \rightarrow \infty$. As an example, we have the following.

Theorem 1.1. Assume that

$$
\sup _{v \in E\left(\lambda_{\ell}\right)} \int_{\Omega} F(x, v) d x<\infty,
$$

where $E\left(\lambda_{\ell}\right)$ is the eigenspace of $\lambda_{\ell}$ and

$$
F(x, t)=\int_{0}^{t} f(x, s) d s .
$$


2 Semilinear problems with bounded nonlinear term

Assume also that if there is a sequence $\left\{u_{k}\right\}$ such that

$$
\begin{gathered}
\left\|P_{\ell} u_{k}\right\| \longrightarrow \infty, \quad\left\|\left(I-P_{\ell}\right) u_{k}\right\| \leq C, \\
2 \int_{\Omega} F\left(x, u_{k}\right) d x \longrightarrow b_{0}, \\
f\left(x, u_{k}\right) \longrightarrow f(x) \quad \text { weakly in } L^{2}(\Omega),
\end{gathered}
$$

where $f(x) \perp E\left(\lambda_{\ell}\right)$ and $P_{\ell}$ is the projection onto $E\left(\lambda_{\ell}\right)$, then

$$
b_{0} \leq\left(f, u_{1}\right)-B_{0},
$$

where $B_{0}=\int_{\Omega} W_{0}(x) d x, W_{0}(x)=\sup _{t}\left[\left(\lambda_{\ell-1}-\lambda_{\ell}\right) t^{2}-2 F(x, t)\right]$, and $u_{1}$ is the unique solution of

$$
-\Delta u-\lambda_{\ell} u=f, \quad u \perp E\left(\lambda_{\ell}\right)
$$

Then (1.1) has at least one solution. In particular, the conclusion holds if there is no sequence satisfying (1.6).

A similar result holds if (1.4) is replaced by

$$
\inf _{v \in E\left(\lambda_{\ell}\right)} \int_{\Omega} F(x, v) d x>-\infty .
$$

In proving these results we will make use of the following theorem [2].

Theorem 1.2. Let $N$ be a closed subspace of a Hilbert space $H$ and let $M=N^{\perp}$. Assume that at least one of the subspaces $M, N$ is finite dimensional. Let $G$ be a $C^{1}$-functional on $H$ such that

$$
\begin{aligned}
& m_{1}:=\inf _{w \in M} \sup _{v \in N} G(v+w)<\infty, \\
& m_{0}:=\sup _{v \in N} \inf _{w \in M} G(v+w)>-\infty .
\end{aligned}
$$

Then there are a constant $c \in \mathbb{R}$ and a sequence $\left\{u_{k}\right\} \subset H$ such that

$$
m_{0} \leq c \leq m_{1}, \quad G\left(u_{k}\right) \longrightarrow c, \quad G^{\prime}\left(u_{k}\right) \longrightarrow 0 .
$$

\section{The main theorem}

We now state our basic result. Let $\Omega$ be a domain in $\mathbb{R}^{n}$, and let $A$ be a selfadjoint operator on $L^{2}(\Omega)$ such that the following hold.

(A)

$$
\sigma_{e}(A) \subset(0, \infty)
$$

(B) There is a function $V(x)>0$ in $L^{2}(\Omega)$ such that multiplication by $V$ is a compact operator from $D:=D\left(|A|^{1 / 2}\right)$ to $L^{1}(\Omega)$.

(C) If $u \in N(A) \backslash\{0\}$, then $u \neq 0$ a.e. in $\Omega$. 
Let $f(x, t)$ be a Carathéodory function on $\Omega \times \mathbb{R}$ satisfying (D)

$$
|f(x, t)| \leq V(x)
$$

Let $\underline{\lambda}(\bar{\lambda})$ be the largest (smallest) negative (positive) point in $\sigma(A)$, and define

$$
\begin{aligned}
& W_{0}(x):=\sup _{t}\left[\underline{\lambda} t^{2}-2 F(x, t)\right], \\
& W_{1}(x):=\sup _{t}\left[2 F(x, t)-\bar{\lambda} t^{2}\right],
\end{aligned}
$$

where

$$
F(x, t):=\int_{0}^{t} f(x, s) d s .
$$

Note that (D) implies

$$
-V(x)^{2} \underline{\lambda} \leq W_{0}(x), \quad W_{1}(x) \leq \frac{V(x)^{2}}{\bar{\lambda}} .
$$

We also assume

(E)

$$
\sup _{v \in N(A)} \int_{\Omega} F(x, v) d x<\infty .
$$

(F) If there is a sequence $\left\{u_{k}\right\} \subset D$ such that

$$
\begin{gathered}
\left\|P_{0} u_{k}\right\| \longrightarrow \infty, \quad\left\|\left(I-P_{0}\right) u_{k}\right\| \leq \text { const } \\
2 \int_{\Omega} F\left(x, u_{k}\right) d x \longrightarrow b_{0}, \quad f\left(x, u_{k}\right) \longrightarrow f(x) \quad \text { weakly in } L^{2}(\Omega),
\end{gathered}
$$

where $f(x) \in R(A)$ and $P_{0}$ is the projection of $D$ onto $N(A)$, then $b_{0} \leq\left(f, u_{1}\right)-B_{0}$, where $B_{0}=\int_{\Omega} W_{0}(x) d x$ and $u_{1}$ is the unique solution of

$$
A u=f, \quad u \in R(A) .
$$

We have the following.

THEOREM 2.1. Under hypotheses $(A)-(F)$, there is at least one solution of

$$
A u=f(x, u), \quad u \in D .
$$

Proof. We begin by letting

$$
N^{\prime}=\oplus_{\lambda<0} N(A-\lambda), \quad N=N^{\prime} \oplus N(A), \quad M=N^{\perp} \cap D, \quad M=M^{\prime} \oplus N(A) .
$$

By hypothesis $(\mathrm{A}), N^{\prime}, N(A)$, and $N$ are finite dimensional, and

$$
D=M \oplus N^{\prime}=M^{\prime} \oplus N
$$


4 Semilinear problems with bounded nonlinear term

It is easily verified that the functional

$$
G(u):=(A u, u)-2 \int_{\Omega} F(x, u) d x
$$

is continuously differentiable on $D$. We take

$$
\|u\|_{D}^{2}:=(|A| u, u)+\left\|P_{0} u\right\|^{2}
$$

as the norm squared on $D$. We have

$$
\left(G^{\prime}(u), v\right)=2(A u, v)-2(f(x, u), v), \quad u, v \in D .
$$

Consequently (2.10) is equivalent to

$$
G^{\prime}(u)=0, \quad u \in D
$$

Note that

$$
\begin{gathered}
(A v, v) \leq \underline{\lambda}\|v\|^{2}, \quad v \in N^{\prime}, \\
\bar{\lambda}\|w\|^{2} \leq(A w, w), \quad w \in M^{\prime} .
\end{gathered}
$$

By hypothesis (D), (2.5), and (2.13),

$$
G(v) \leq \underline{\lambda}\|v\|^{2}+2\|V\| \cdot\|v\| \longrightarrow-\infty \quad \text { as }\|v\| \longrightarrow \infty, v \in N^{\prime} .
$$

For $w \in M$, we write $w=y+w^{\prime}, y \in N(A), w^{\prime} \in M^{\prime}$. Since $|F(x, w)-F(x, y)| \leq V(x)\left|w^{\prime}\right|$ by (D) and (2.5), we have

$$
G(w) \geq \bar{\lambda}\left\|w^{\prime}\right\|^{2}-2 \int F(x, y) d x-2\|V\| \cdot\left\|w^{\prime}\right\| .
$$

In view of (E), (2.19) and (2.20) imply

$$
\inf _{M} G>-\infty, \quad \sup _{N^{\prime}} G<\infty .
$$

We can now apply Theorem 1.2 to conclude that there is a sequence satisfying (1.11). Let

$$
u_{k}=v_{k}+w_{k}+\rho_{k} y_{k}, \quad v_{k} \in N^{\prime}, w_{k} \in M^{\prime}, y_{k} \in N(A),\left\|y_{k}\right\|=1, \rho_{k} \geq 0
$$

We claim that

$$
\left\|u_{k}\right\|_{D} \leq C
$$

To see this, note that (1.11) and (2.15) imply

$$
\left(A u_{k}, h\right)-\left(f\left(x, u_{k}\right), h\right)=o(\|h\|) .
$$


Taking $h=v_{k}$, we see that $\left\|v_{k}\right\|^{2}=O\left(\left\|v_{k}\right\|\right)$ in view of (2.17) and (D). Thus $\left\|v_{k}\right\|_{D}$ is bounded. Similarly, taking $h=w_{k}$, we see that $\left\|w_{k}\right\|_{D} \leq C$. Suppose

$$
\rho_{k} \longrightarrow \infty
$$

There is a renamed subsequence such that $y_{k} \rightarrow y$ in $N(A)$. Clearly $\|y\|=1$. Thus by hypothesis (D), $y \neq 0$ a.e. This means that $\left\|\rho_{k} y_{k}\right\| \rightarrow \infty$. Hence (2.8) holds. Let $u_{k}^{\prime}=v_{k}+$ $w_{k} \in N(A)^{\perp}=R(A)$. Then $\left\|u_{k}^{\prime}\right\|_{D} \leq C$. Thus there is a renamed subsequence such that $u_{k}^{\prime} \rightarrow u_{1}$ weakly in $D$. By hypothesis (B), there is a renamed subsequence such that $V u_{k}^{\prime} \rightarrow$ $V u_{1}$ strongly in $L^{1}(\Omega)$. Since $V(x)>0$, there is another renamed subsequence such that $u_{k}^{\prime} \rightarrow u_{1}$ a.e. in $\Omega$. On the other hand, since $f_{k}(x)=f\left(x, u_{k}(x)\right)$ is uniformly bounded in $L^{2}(\Omega)$ by hypothesis $(\mathrm{D})$, there is an $f(x) \in L^{2}(\Omega)$ such that for a subsequence

$$
f_{k}(x) \longrightarrow f(x) \text { weakly in } L^{2}(\Omega) .
$$

Since

$$
\left(A u_{k}^{\prime}, h\right)-\left(f_{k}(x), h\right)=o\left(\|h\|_{D}\right), \quad h \in D
$$

we see in the limit that $u_{1}$ is a solution of (2.9), and consequently that $f \in R(A)$. Moreover, we see by (2.27) that

$$
\left(A\left[u_{k}^{\prime}-u_{1}\right], h\right)-\left(f_{k}-f, h\right)=o\left(\|h\|_{D}\right), \quad h \in D .
$$

Write $u_{1}=v_{1}+w_{1}$, and take $h$ successively equal to $v_{k}-v_{1}$ and $w_{k}-w_{1}$. Then

$$
\begin{gathered}
\left\|v_{k}-v_{1}\right\|_{D}^{2} \leq 2\left\|V\left[v_{k}-v_{1}\right]\right\|_{1}+o\left(\left\|v_{k}^{\prime}-v_{1}\right\|_{D}\right), \\
\left\|w_{k}-w_{1}\right\|_{D}^{2} \leq 2\left\|V\left[w_{k}-w_{1}\right]\right\|_{1}+o\left(\left\|w_{k}-w_{1}\right\|_{D}\right) .
\end{gathered}
$$

Hence $u_{k}^{\prime} \rightarrow u_{1}$ in $D$. Consequently,

$$
\begin{gathered}
\left(A u_{k}, u_{k}\right)=\left(A u_{k}^{\prime}, u_{k}^{\prime}\right)=\left(f_{k}, u_{k}^{\prime}\right)+o\left(\left\|u_{k}^{\prime}\right\|\right) \longrightarrow\left(f, u_{1}\right), \\
2 \int F\left(x, u_{k}\right) d x=\left(A u_{k}, u_{k}\right)-G\left(u_{k}\right) \longrightarrow\left(f, u_{1}\right)-c,
\end{gathered}
$$

where $m_{0} \leq c \leq m_{1}$. By $(2.3)$

$$
G(v) \leq(A v, v)-\underline{\lambda}\|v\|^{2}+B_{0}, \quad v \in N^{\prime} .
$$

Thus $m_{1} \leq B_{0}$. Consider first the case $m_{1}<B_{0}$. Then (2.31) implies $b_{0}=\left(f, u_{1}\right)-c$, and consequently, $m_{0} \leq\left(f, u_{1}\right)-b_{0} \leq m_{1}<B_{0}$. Thus $b_{0}>\left(f, u_{1}\right)-B_{0}$, contradicting (1.7). This shows that the assumption (2.25) is not possible. Consequently (2.23) holds, and we have a renamed subsequence such that $u_{k} \rightarrow u$ strongly in $D$ and a.e. in $\Omega$. It now follows from (2.27) that

$$
(A u, h)=(f(x, u), h), \quad h \in D
$$


6 Semilinear problems with bounded nonlinear term

showing that (2.10) indeed has a solution. Assume now that $m_{1}=B_{0}$. Let $v_{k}$ be a maximizing sequence in $N^{\prime}$ such that $G\left(v_{k}\right) \rightarrow m_{1}$. By (2.19), $\left\|v_{k}\right\|_{D} \leq C$, and there is a renamed subsequence such that $v_{k} \rightarrow v_{0}$ in $N^{\prime}$. By continuity $G\left(v_{k}\right) \rightarrow G\left(v_{0}\right)$. Hence $G\left(v_{0}\right)=m_{1}=$ $B_{0}$. Thus

$$
\underline{\lambda}\left\|v_{0}\right\|^{2} \leq 2 \int F\left(x, v_{0}\right) d x+B_{0}=\left(A v_{0}, v_{0}\right) \leq \underline{\lambda}\|v\|^{2} .
$$

Consequently, $\left(A v_{0}, v_{0}\right)=\underline{\lambda}\left\|v_{0}\right\|^{2}$ and $A v_{0}=\underline{\lambda} v_{0}$. We also have

$$
\int_{\Omega}\left[2 F\left(x, v_{0}\right)-\underline{\lambda} v_{0}^{2}+W_{0}(x)\right] d x=0 .
$$

In view of (2.3), the integrand is nonnegative. Hence

$$
2 F\left(x, v_{0}\right) \equiv \underline{\lambda} v_{0}^{2}-W_{0}(x)
$$

Let

$$
\Phi(u)=\int_{\Omega}\left[2 F(x, u)-\underline{\lambda} u^{2}\right] d x
$$

Then

$$
\begin{gathered}
\Phi(u) \geq \Phi\left(v_{0}\right), \quad u \in D, \\
\left(\Phi^{\prime}(u), y\right)=2(f(x, u), h)-2 \underline{\lambda}(u, h) .
\end{gathered}
$$

Thus

$$
\Phi^{\prime}\left(v_{0}\right)=2 f\left(x, v_{0}\right)-2 \underline{\lambda} v_{0} \equiv 0 \text {. }
$$

This implies

$$
A v_{0}=\underline{\lambda} v_{0}=f\left(x, v_{0}\right)
$$

and $v_{0}$ is a solution of (2.10). This completes the proof.

Theorem 2.2. In Theorem 2.1, replace hypotheses (E), (F) by

(E')

$$
\inf _{v \in N(A)} \int_{\Omega} F(x, v) d x>-\infty,
$$

(F') if (2.8) hold with $f(x) \in R(A)$, then

$$
b_{0} \geq\left(f, u_{1}\right)+B_{1} .
$$

Then (2.10) has at least one solution. 
Proof. We modify the proof of Theorem 2.1. This time we use the second decomposition in (2.12). For $v \in N$ we write $v=v^{\prime}+v_{0}$, where $v^{\prime} \in N^{\prime}$ and $v_{0} \in N(A)$. By (D) and (2.5),

$$
\int_{\Omega} F\left(x, v_{0}\right) d x \leq \int_{\Omega} F(x, v) d x+\|V\| \cdot\left\|v^{\prime}\right\| .
$$

Hence

$$
G(v) \leq \underline{\lambda}\left\|v^{\prime}\right\|^{2}+2\|V\| \cdot\left\|v^{\prime}\right\|-2 \int F\left(x, v_{0}\right) d x, \quad v \in N .
$$

Consequently,

$$
m_{1}=\sup _{N} G<\infty
$$

On the other hand

$$
G(w) \geq \bar{\lambda}\|w\|^{2}-2\|V\| \cdot\|w\|, \quad w \in M^{\prime}
$$

so that

$$
m_{0}=\inf _{M^{\prime}} G>-\infty .
$$

It now follows from Theorem 1.2 that there is a sequence $\left\{u_{k}\right\} \subset D$ satisfying (1.11). We now follow the proof of Theorem 2.1 from (2.22) to (2.31). By (2.4),

$$
G(w) \geq(A w, w)=\bar{\lambda}\|w\|^{2}-B_{1}, \quad w \in M^{\prime},
$$

where $B_{1}=\int_{\Omega} W_{1}(x) d x$. Thus $m_{0} \geq-B_{1}$. Assume first that $m_{0}>-B_{1}$. Then (1.11) and (2.31) imply

$$
-B_{1}<m_{0} \leq\left(f, u_{1}\right)-b_{0}
$$

contradicting (2.42). Thus (2.25) cannot hold, and we obtain a solution of (2.10) as in the proof of Theorem 2.1. If $m_{0}=-B_{1}$, let $\left\{w_{k}\right\} \subset M^{\prime}$ be a minimizing sequence such that $w_{k} \rightarrow w_{0}$ weakly in $D, V w_{k} \rightarrow V w_{0}$ in $L^{1}(\Omega)$ and a.e. in $\Omega$. By hypothesis (D),

$$
\int_{\Omega}\left[F\left(x, w_{k}\right)-F\left(x, w_{0}\right)\right] d x=\int_{\Omega} \int_{0}^{1} f\left(x, w_{0}+\theta\left(w_{k}-w_{0}\right)\right)\left(w_{k}-w_{0}\right) d \theta d x \longrightarrow 0
$$

Thus $G$ is weakly lower semicontinuous, and

$$
G\left(w_{0}\right) \leq \lim G\left(w_{k}\right)=m_{0}-B_{1} .
$$

Hence

$$
\bar{\lambda} w_{0}=f\left(x, w_{0}\right) \leq 2 \int F\left(x, w_{0}\right)-B_{1} \leq \bar{\lambda}\left\|w_{0}\right\|^{2}
$$


8 Semilinear problems with bounded nonlinear term

and we proceed as before to show that

$$
A w_{0}=\bar{\lambda} w_{0}=f\left(x, w_{0}\right) .
$$

The proof is complete.

\section{References}

[1] E. M. Landesman and A. C. Lazer, Nonlinear perturbations of linear elliptic boundary value problems at resonance, J. Math. Mech. 19 (1969/1970), 609-623.

[2] M. Schechter, A generalization of the saddle point method with applications, Ann. Polon. Math. 57 (1992), no. 3, 269-281.

Martin Schechter: Department of Mathematics, University of California, Irvine, CA 92697-3875, USA

E-mail address: mschecht@math.uci.edu 